\title{
ORIGINAL ARTICLE \\ Comparison of bacterial strains and antibiotic susceptibilities in urinary isolates of spinal cord injury patients from the community and hospital
}

\author{
SB Yoon ${ }^{1}$, BS Lee ${ }^{2}$, KD Lee ${ }^{1}$, SI Hwang ${ }^{3}, \mathrm{HJ} \mathrm{Lee}^{2}$ and ZA Han ${ }^{2}$
}

\begin{abstract}
Study design: A one-year epidemiological survey.
Objective: To compare bacterial strains and antimicrobial susceptibilities of urinary isolates from hospital and community spinal cord injury $(\mathrm{SCl})$ patients.

Setting: A specialized $\mathrm{SCl}$ unit in a freestanding rehabilitation hospital.

Methods: From June 2012 through May 2013, urine cultures were obtained from all of the newly admitted patients. Bacterial strains and antimicrobial susceptibilities were compared between patients from community and hospital settings.

Results: The proportion of Enterobacteriaceae in the total urinary isolates from hospital-dwelling patients was smaller than that from community-dwelling patients (66.0 vs $85.5 \%, P<0.001)$, while the proportions of Pseudomonas, Acinetobacter and Enterococcus species were relatively larger $(8.7 \%, 6.0 \%$ and $12.0 \%$ vs $2.8 \%, 0.7 \%$ and $2.8 \%$, respectively, $P<0.05)$. The isolates from hospitaldwelling patients showed lower susceptibility to ampicillin, amoxicillin-clavulanic acid, trimethoprim-sulfamethoxazole and all generations of cephalosporin $(P<0.05)$, and a higher prevalence of extended-spectrum beta-lactamase $(\mathrm{ESBL})$-producers $(41.7$ vs $5.4 \%, P<0.001)$, compared with those from community-dwelling patients. The susceptibility rates to levofloxacin were lower than $50 \%$ in both community and hospital-dwelling patients.

Conclusion: Broader-spectrum antibiotics should be considered in treating nosocomial urinary tract infection (UTI) of SCl patients because of the relatively wide variety of organisms and higher frequency of antibiotic-resistant strains, including ESBL-producing Enterobacteriaceae in hospital-derived specimens. Furthermore, in areas with high prevalence of fluoroquinolone resistance, fluoroquinolones should be used with caution during empirical treatment for UTI in SCI patients.
\end{abstract}

Spinal Cord (2014) 52, 298-301; doi:10.1038/sc.2014.10; published online 11 February 2014

Keywords: spinal cord injuries; urinary tract infections; bacteria; antibacterial sensitivity tests

\section{INTRODUCTIONS}

People with spinal cord injury (SCI) have increased risk of developing urinary tract infections (UTIs). The use of catheters, elevated bladder pressure, and increased post-void residual volumes are the major contributing factors for UTI in these patients. ${ }^{1,2}$ Although long-term urologic rehabilitation has advanced, UTI is still one of the leading cause of morbidity in patients with SCI, and is also associated with a significantly increased mortality. ${ }^{3}$ UTI is a clinical challenge for both patients and physicians.

UTI has also been known to be the most common type of nosocomial infection in SCI patients. ${ }^{4}$ SCI patients have long hospital stays and such frequent and prolonged hospitalization can affect bacterial strains in urine, and can increase the risk of acquiring antibiotic resistance. These changes may lead to inappropriate antibiotic selection and treatment failure.

Some previous studies have investigated the spectrum of microorganisms and their antibacterial susceptibilities from urinary tract isolates of SCI patients. ${ }^{5-7}$ The results of these studies may help to select proper antimicrobial agents during empirical therapy for UTI. However, these studies have only assessed the outpatient SCI population.
Empiric antibiotic treatment for UTI should be initiated on the basis of knowledge of the local bacterial ecology. In this regard, identification of pathogens and assessing their susceptibility to antimicrobial agents in relation to areas of residence is essential for implementing proper empirical treatment. We compared the bacterial strains of urinary isolates from hospital and community SCI patients. We also analyzed the antibiotic susceptibility profile of Enterobacteriaceae, which is known to be the major uropathogen in SCI patients. ${ }^{2,8}$

This study is the first to investigate and compare urine specimens of both community and hospital-residing SCI patients. Such an evaluation can be useful for establishing antibiotic policy on empirical UTI treatment in SCI patients.

\section{MATERIALS AND METHODS \\ Setting and design}

This study was performed at a specialized SCI unit at National Rehabilitation Center of Korea. The National Rehabilitation Center is specialized for rehabilitation treatment and related services and is a referral hospital that accepts patients from other hospitals throughout the nation. It also provides

${ }^{1}$ Department of Internal Medicine, National Rehabilitation Center, Seoul, Korea; ${ }^{2}$ Department of Spinal Cord Injury Rehabilitation, National Rehabilitation Center, Seoul, Korea and ${ }^{3}$ Department of Rehabilitation, Soonchunhyang University Hospital, Seoul, Korea

Correspondence: Dr ZA Han, Department of Spinal Cord Medicine Injury Rehabilitation, National Rehabilitation Center, 58, Samgaksan-ro, Gangbuk-gu, Seoul 142-884, Korea. E-mail: zeeahan@catholic.ac.kr

Received 10 September 2013; revised 6 January 2014; accepted 13 January 2014; published online 11 February 2014 
annual check-up programs on urologic function for SCI patients from the community. In the rehabilitation center, urine cultures were routinely requested on admission to screen for the presence of highly resistant microorganism and determine the need for active surveillance and isolation.

This epidemiological survey was carried out for one year, between June 2012 and May 2013. Urine samples were obtained on admission from all SCI patients during the study period. We collected data from electronic patient files including demographic characteristics, etiology and date of injury, injury level, voiding method and results of urine cultures. There were no missing values in the data set. This study was approved by the Institutional Review Board of this study institution. The written informed consents were exempted by the board because this study was performed assessing the data of patients that had been obtained during standard medical care.

\section{Patients}

SCI patients over the age of 18 years who were admitted to the National Rehabilitation Center during the study period were included in this study. The patients were divided into two groups with one being community-residing SCI patients who were undergoing their annual urologic function check. SCI patients who had not been hospitalized within 90 days before admission were defined as community-residing SCI patients on the basis of definitions given in a previous study. ${ }^{9}$ The other group comprised hospital-dwelling SCI patients who were transferred to our institute for intensive rehabilitation from other hospitals.

\section{Urine cultures}

Urine samples were collected from all patients on admission day. A clean-catch midstream technique was used for patients who were able to void. Meanwhile, urine samples were collected sterilely from the bladder at the time of intermittent catheterization, or from indwelling catheters for patients who were incapable of voluntary bladder voiding in accordance with guidelines established by the center. All specimens were cultured and isolates were identified at the authorized institute (Seoul Clinical Laboratories, Seoul, Korea).

Significant bacteriuria was defined as presenting $10^{4}$ or more colonyforming units per $\mathrm{ml}$. Bacterial identification and antibiotic susceptibility testing were performed using Vitek 2 system (bioMérieux, Durham, NC, USA). Antibiotic susceptibility for ampicillin, amoxicillin-clavulanic acid, cefazolin, cefuroxime, cefotaxime, cefepime, trimethoprim-sulfamethoxazole, levofloxacin, gentamycin, amikacin and imipenem were tested for strains in the Enterobacteriaceae family. Screening test for extended-spectrum beta-lactamase (ESBL) was also conducted for Escherichia coli and Klebsiella species. Antibiotic susceptibility and the presence of ESBL from grown isolates were determined according to the Clinical and Laboratory Standards Institute guidelines. ${ }^{10}$

\section{Statistical analysis}

Continuous data are presented as means \pm s.d., and categorical data as quantities and proportions. A Chi-square test or a Fisher's exact test was used to analyze categorical data and the two-sample independent $t$-test was used to analyze continuous data. Factors potentially associated with antibiotic resistance were investigated using univariate and multivariate logistic regression analyses. We performed a regression analysis on second-generation cephalosporin (cefuroxime) on the basis that it is an antibiotic generally used in the empirical treatment of UTI, ${ }^{3}$ and most commonly used at our institution. The analysis was performed using STATA version 12.1 (StataCorp., College Station, TX, USA), and statistical significance was accepted for $P$-values of $<0.05$.

\section{RESULTS}

A total of 424 patients were included in the study. The mean age of the study group was $48.3 \pm 14.7$ and $73.1 \%$ were male. Out of the total, 189 patients were community residing and 235 were those transferred from other hospitals. The baseline characteristics of the two groups are summarized in Table 1.

The most common microorganisms detected in the urine cultures in both community and hospital-dwelling SCI patients were the Enterobacteriaceae family (Table 2). However, the proportion of Enterobacteriaceae among the total urinary isolates of hospital-dwelling patients was smaller than that of the community-dwelling patients ( 66.0 vs $85.5 \%, P<0.001$ ). Meanwhile, the proportions of Pseudomonas, Acinetobacter and Enterococcus species were relative larger in the hospital-residing patients, compared with those from the community $(8.7 \%, 6.0 \%$ and $12.0 \%$ vs $2.8 \%, 0.7 \%$ and $2.8 \%$, respectively, $P<0.05)$. E. coli and Klebsiella species were the first and the second most common strains, respectively, in the Enterobacteriaceae family of both community and hospital-dwelling patients, and these two species comprised $70 \%$ of the total Enterobacteriaceae family (Table 3).

Table 1 Clinical characteristics of the patients from community and hospital $(N=424)$

\begin{tabular}{llcc}
\hline & & Community & Hospital \\
& & $(\mathrm{n}=189)$ & $(\mathrm{n}=235)$ \\
\hline Age (years) & Mean \pm s.d. (range) & $45.2 \pm 12.7$ & $50.9 \pm 15.7$ \\
& & $(18-78)$ & $(19-84)$ \\
Sex & Male (\%) & $146(77.2 \%)$ & $164(69.8 \%)$ \\
Duration of injury & Mean \pm s.d. (range) & $126.5 \pm 97.5$ & $12.5 \pm 22.3$ \\
(months) & & $(3-532)$ & $(1-164)$ \\
Etiology of injury & Trauma (\%) & $160(84.7 \%)$ & $180(76.6 \%)$ \\
& Non-trauma (\%) & $29(15.3 \%)$ & $55(23.4 \%)$ \\
Injury level & Paraplegia (\%) & $104(55.0 \%)$ & $106(45.1 \%)$ \\
Voiding method & Tetraplegia (\%) & $85(45.0 \%)$ & $129(54.9 \%)$ \\
& Spontaneous voiding (\%) & $35(18.5 \%)$ & $73(31.1 \%)$ \\
Cic (\%) & $125(66.1 \%)$ & $78(33.2 \%)$ \\
Number of & Indwelling catheter (\%) & $29(15.3 \%)$ & $84(35.7 \%)$ \\
uropathogens & None (\%) & $52(27.5 \%)$ & $89(37.9 \%)$ \\
& & & \\
& Single organism (\%) & $133(70.4 \%)$ & $142(60.4 \%)$ \\
& Double organisms (\%) & $4(2.1 \%)$ & $4(1.7 \%)$ \\
\hline
\end{tabular}

Abbreviation: CIC, clean intermittent catheterization.

Table 2 Microorganisms of urinary isolates in patients from community and hospital $(N=291)$

\begin{tabular}{|c|c|c|c|}
\hline & $\begin{array}{l}\text { Community } \\
(\mathrm{n}=141)\end{array}$ & $\begin{array}{l}\text { Hospital } \\
(\mathrm{n}=150)\end{array}$ & P-value \\
\hline \multicolumn{4}{|l|}{ Gram-negative species } \\
\hline Enterobacteriaceae family & $121(85.5 \%)$ & $99(66.0 \%)$ & $<0.001$ \\
\hline Pseudomonas species & $4(2.8 \%)$ & $13(8.7 \%)$ & 0.044 \\
\hline Acinetobacter species & $1(0.7 \%)$ & $9(6.0 \%)$ & $0.020^{a}$ \\
\hline \multicolumn{4}{|l|}{ Gram-positive species } \\
\hline Enterococcus species & $4(2.8 \%)$ & $18(12.0 \%)$ & 0.003 \\
\hline Staphylococcus species & $4(2.8 \%)$ & $3(2.0 \%)$ & $\mathrm{NS}^{\mathrm{a}}$ \\
\hline Streptococcus species & $3(2.1 \%)$ & $2(1.7 \%)$ & $\mathrm{NS}^{\mathrm{a}}$ \\
\hline Others & $4^{b}(2.8 \%)$ & $6^{c}(3.0 \%)$ & $\mathrm{NS}^{\mathrm{a}}$ \\
\hline \multicolumn{4}{|c|}{$\begin{array}{l}\text { Abbreviation: NS, not-significant. } \\
\text { aFisher's exact tests were used. } \\
\text { 'Burkholderia capacia (1), Flavabacterium ordoratum (1), Stenotrophomonas maltophilia (1) } \\
\text { and undefined Gram-positive bacillus (1). } \\
\text { 'Flavabacterium ordoratum (1), Stenotrophomonas maltophilia (1), Alcaligenes xylosoxidans (1) } \\
\text { and Yeasts (3). }\end{array}$} \\
\hline
\end{tabular}


The susceptibility rates of Enterobacteriaceae to various antimicrobial agents, and the prevalence of ESBL-producers in E. coli and Klebsiella species are presented in Figure 1. The isolates from hospitaldwelling patients showed significantly lower susceptibility to ampicillin, amoxicillin-clavulanic acid, trimethoprim-sulfamethoxazole

Table 3 Bacterial strains of Enterobacteriaceae family in patients from community and hospital $(N=220)$

\begin{tabular}{lccc}
\hline & Community $(\mathrm{n}=121)$ & Hospital $(\mathrm{n}=99)$ & Total $(\mathrm{N}=220)$ \\
\hline Escherichia coli & $73(60.3 \%)$ & $44(44.4 \%)$ & $117(53.2 \%)$ \\
Klebsiella species & $19(15.7 \%)$ & $28(28.3 \%)$ & $47(19.1 \%)$ \\
Serratia marcescens & $11(9.1 \%)$ & $9(9.1 \%)$ & $20(9.1 \%)$ \\
Enterobacter species & $5(4.1 \%)$ & $5(5.1 \%)$ & $10(4.5 \%)$ \\
Citrobacter species & $5(4.1 \%)$ & $3(3.0 \%)$ & $8(3.6 \%)$ \\
Proteus mirabilis & $4(3.3 \%)$ & $3(3.0 \%)$ & $7(3.2 \%)$ \\
Morganella morganii & $2(1.7 \%)$ & $5(5.1 \%)$ & $7(3.2 \%)$ \\
Providencia species & $2(1.7 \%)$ & $1(1.0 \%)$ & $3(1.4 \%)$ \\
Cedecea davisae & $0(0.0 \%)$ & $1(1.0 \%)$ & $1(0.5 \%)$ \\
\hline
\end{tabular}

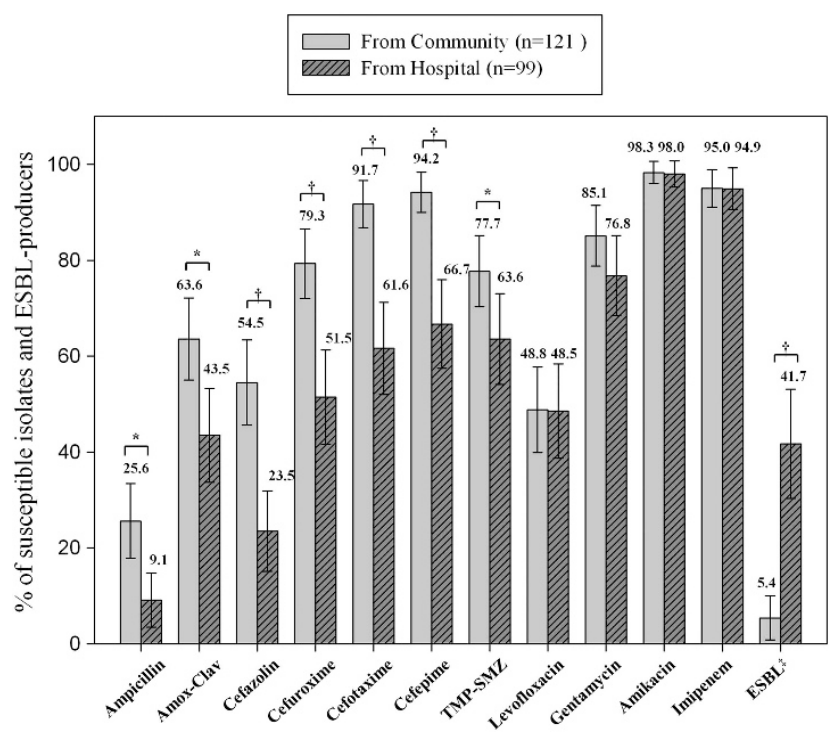

Figure 1 The antibiotic susceptibility rates for Enterobacteriaceae and the prevalence of ESBL-producers. Amox-Clav, amoxicillin-clavulanic acid; TMP-SMZ, trimethoprim-sulfamethoxazole. ${ }^{*} P<0.05$. $\dagger P<0.001$. †The screening test and prevalence calculation for ESBL were conducted only in Escherichia coli and Klebsiella species. and all generations of cephalosporin, compared with those from community-dwelling patients $(P<0.05)$. Amikacin and imipenem were the most effective antibiotics with more than $90 \%$ of the isolates being susceptible to these antibiotics in both groups. Meanwhile, the isolates were less susceptible to levofloxacin, which showed lower than $50 \%$ susceptibility rates regardless of residency status. The prevalence of ESBL-producers in E. coli and Klebsiella species was significantly higher in isolates from hospital-dwelling patients than those from community-dwelling patients ( 41.7 vs $5.4 \%, P<0.001$ ).

Logistic regression results for factors associated with cefuroxime resistance are summarized in Table 4. Compared with communityresiding patients, odds ratios for cefuroxime resistance of hospitalresiding patients was 3.61 (95\% confidence intervals (CI), 2.00-6.53) and 3.19 (95\% CI, 1.68-6.02) in univariate and multivariate analysis, respectively. Age, sex, injury level and voiding methods were also investigated as factors potentially associated with the resistance. However, both univariate and multivariate regression analyses did not show an association between these factors and antibiotic resistance.

\section{DISCUSSION}

This study showed that bacterial strains and their antibiotic susceptibilities of urinary isolates were different between SCI patients from the community and those from the hospital. Various nosocomial organisms, such as Pseudomonas, Acinetobacter and Enterococcus species, were more frequently observed in hospital-dwelling patients. In addition, microorganisms in the Enterobacteriaceae family from hospital-dwelling patients were less susceptible to various antimicrobial agents, and were at high risk for becoming ESBL-producers.

The Enterobacteriaceae family was the major urine isolates in both SCI patient groups. However considering that the proportions of Pseudomonas, Acinetobacter and Enterococcus were higher in the nosocomial group, such isolates should also be considered as possible uropathogens especially in hospitalized SCI patients. Pseudomonas and Acinetobacter species have already been known as established pathogens in general hospital-acquired infections, and the treatment of these species is a challenging task because of their limited susceptibility and the emergence of resistance during therapy. ${ }^{11,12}$ Enterococcus species can also be possible UTI pathogens in hospitalized SCI patients, and have been known to be relatively more common in catheter-associated UTI. ${ }^{8}$ Because of their inherent resistance to cephalosporin and aminoglycoside, specialized antimicrobial management should be considered in treating Enterococcal UTI. ${ }^{13}$

Besides the wide variety of organisms, degree of antimicrobialresistance was different between the two SCI patient groups. In SCI

Table 4 Logistic regression analyses for factors associated with the resistance of Enterobacteriaceae to second-generation cephalosporin (cefuroxime)

\begin{tabular}{|c|c|c|c|c|}
\hline \multirow[t]{2}{*}{ Factor } & \multicolumn{2}{|c|}{ Univariate analysis } & \multicolumn{2}{|c|}{ Multivariate analysis } \\
\hline & OR $(95 \% \mathrm{Cl})$ & $\mathrm{P}$-value & OR $(95 \% \mathrm{Cl})$ & P-value \\
\hline Age $\geqslant 60$ years (vs $<60$ years) & $1.49(0.85-2.61)$ & 0.167 & $1.29(0.69-2.41)$ & 0.418 \\
\hline Male (vs female) & $1.44(0.78-2.64)$ & 0.242 & $1.99(0.99-3.99)$ & 0.053 \\
\hline Tetraplegia (vs paraplegia) & $1.04(0.59-1.83)$ & 0.886 & $0.80(0.42-1.51)$ & 0.488 \\
\hline CIC (vs spontaneous voiding) & $0.46(0.20-1.05)$ & 0.064 & $0.49(0.21-1.17)$ & 0.109 \\
\hline Indwelling catheter (vs spontaneous voiding) & $1.67(0.72-3.88)$ & 0.237 & $1.43(0.57-1.51)$ & 0.441 \\
\hline From hospital (vs from community) & $3.61(2.00-6.53)$ & $<0.001$ & $3.19(1.68-6.02)$ & $<0.001$ \\
\hline
\end{tabular}

Abbreviations: $\mathrm{Cl}$, confidence interval; $\mathrm{CIC}$, clean intermittent catheterization; OR, odds ratio. 
patients, not only hospital but also community-residing patients have problems associated with antibiotic-resistant bacterial colonization or infection due to catheter-associated voiding and frequent antibiotics usage. ${ }^{5,6,14}$ However, compared with community-dwelling patients, isolates from hospital-dwelling SCI patients showed higher resistance rates to the majority of antimicrobial agents that were investigated. In related studies, trimethoprim-sulfamethoxazole, amoxicillinclavulanic acid and second or third generation of cephalosporins are generally recommended for the empirical treatment of UTI in patients with SCI.,8 However, in our study, these recommended antimicrobial agents were shown to be less susceptible in isolates from hospital-residing SCI patients. Therefore, careful clinical consideration should be made when empirically treating nosocomial UTI of SCI patient.

ESBL has been known as the most relevant determinant of resistance in Enterobacteriaceae. In our study, the prevalence of ESBL-producers from hospital-dwelling patients was about seven times higher than that from community-dwelling patients, and comprised more than $40 \%$ in E. coli and Klebsiella species. ESBLproducing organisms not only are capable of degrading the extendedspectrum cephalosporins and monobactams, but also often exhibit cross-resistance to other types of antibiotics, such as fluoroquinolones and aminoglycosides. ${ }^{15}$ In addition, infections with ESBL-producing strains have a significant impact on increased morbidity, mortality and health care-associated costs. ${ }^{16}$ Carbapenem should be regarded as the drug of choice when treating serious UTI suspicious by ESBLproducing organisms in hospital-dwelling SCI patients.

Contrary to expectation, susceptibility to levofloxacin was not different according to areas of residence in the study. Surprisingly the susceptibility rate was lower than $50 \%$ even in the pathogens from community-dwelling patients. Fluoroquinolones are the most frequently used medications for UTI, because they provide a broad spectrum of antimicrobial activity, achieve high levels in the urinary tract tissue, and can be easily administered orally. ${ }^{17}$ However, due to their excessive use, an increase of resistance to fluoroquinolones is becoming a major threat in Korea. ${ }^{18}$ In areas with high prevalence of fluoroquinolone resistance, this drug should not be recommended for empirical use in UTI of SCI patients.

This study has several limitations. First, this study assessed bacteriuria, not symptomatic UTI. Bacteriuria in itself does not necessary imply true infection, because it may indicate mere colonization and not bacteria with actual tissue invasion. ${ }^{19}$ However, once colonization of the urinary tract occurs, the SCI patient is probably at higher risk for further tissue invasion and symptomatic UTI by the colonizing organism. A further comparison study assessing symptomatic UTI between community and hospitaldwelling patients is needed to elaborate and confirm the findings of our study. Second, the comparison of antimicrobial susceptibility was only made in Enterobacteriaceae family. Although antibiotic susceptibility testing was also performed in other species, such as Pseudomonas, Acinetobacter and Enterococcus, we could not compare the antibiotic susceptibility according to patient dwelling due to the minimal number of existing cases. Third, we were unable to confirm hospitalization durations and antibiotic usage in admissions prior to our institution. Thus, analysis of antibiotic sensitivities related to these factors could not be made in this study. Finally, this study was conducted in a single center. However, this institute is a central national rehabilitation agency that accepts nationwide SCI patient referrals and thus can be a reflection of the SCI patients in Korea.

General recommendations for empirical treatment for UTI are not possible because of the wide variety of organisms and their antibiotic susceptibility. ${ }^{20}$ Therefore regimens for empirical treatment must be on the basis of the statistics of the respective center. However, the findings of our study could suggest that, in general, broader-spectrum antibiotics should be considered for use in nosocomial UTI of SCI patients. Especially, ESBL-producing strains have to be considered as possible uropathogens in hospital-dwelling patients. Meanwhile, clinicians should refrain from using fluoroquinolones during empirical UTI treatment in areas known to have high prevalence of its resistance. Furthermore, appropriate duration of antibiotic therapy should be chosen on an individual basis to minimize further resistance. These evidence-based recommendations could be helpful in selecting empirical antibiotics in UTI of SCI patients.

\section{DATA ARCHIVING}

There were no data to deposit.

\section{CONFLICT OF INTEREST}

The authors declare no conflict of interest.

1 Cardenas DD, Hooton TM. Urinary tract infection in persons with spinal cord injury. Arch Phys Med Rehabil 1995; 76: 272-280.

2 Montgomerie JZ. Infections in patients with spinal cord injuries. Clin Infect Dis 1997; 25: 1285-1290.

3 Biering-Sorensen F, Bagi P, Hoiby N. Urinary tract infections in patients with spinal cord lesions: treatment and prevention. Drugs 2001; 61: 1275-1287.

4 Mylotte JM, Graham R, Kahler L, Young L, Goodnough S. Epidemiology of nosocomial infection and resistant organisms in patients admitted for the first time to an acute rehabilitation unit. Clin Infect Dis 2000; 30: 425-432.

5 Waites KB, Chen Y, DeVivo MJ, Canupp KC, Moser SA. Antimicrobial resistance in gram-negative bacteria isolated from the urinary tract in community-residing persons with spinal cord injury. Arch Phys Med Rehabil 2000; 81: 764-769.

6 Hinkel A, Finke W, Botel U, Gatermann SG, Pannek J. Increasing resistance against antibiotics in bacteria isolated from the lower urinary tract of an outpatient population of spinal cord injury patients. Urol Int 2004; 73: 143-148.

7 Martins CF, Bronzatto E, Neto JM, Magalhaes GS, D'Anconna CA, Cliquet Jr A. Urinary tract infection analysis in a spinal cord injured population undergoing rehabilitationhow to treat? Spinal Cord 2012; 51: 193-195.

8 Garcia Leoni ME, Esclarin De Ruz A. Management of urinary tract infection in patients with spinal cord injuries. Clin Microbiol Infect 2003; 9: 780-785.

9 Aguilar-Duran S, Horcajada JP, Sorli L, Montero M, Salvadó M, Grau S et al. Community-onset healthcare-related urinary tract infections: comparison with community and hospital-acquired urinary tract infections. J Infect 2012; 64: 478-483.

10 Clinical and Laboratory Standards Institute. Performances Standards for Antimicrobial Susceptibility Testing; 21st Informational Supplement M100-S21. CLSI: Wayne, PA, USA, 2011.

11 Lombardi G, Musco S, Celso M, lerardi A, Nelli F, Del Corso F et al. A retrospective study on female urological surgeries over the 10 years following spinal cord lesion. Spinal Cord 2013; 51: 688-693.

12 Carmeli Y, Troillet N, Eliopoulos GM, Samore MH. Emergence of antibiotic-resistant Pseudomonas aeruginosa: comparison of risks associated with different antipseudomonal agents. Antimicrob Agents Chemother 1999; 43: 1379-1382.

13 Murray BE. The life and times of the Enterococcus. Clin Microbiol Rev 1990; 3: 46-65.

14 Roghmann MC, Wallin MT, Gorman PH, Johnson JA. Prevalence and natural history of colonization with fluoroquinolone-resistant gram-negative bacilli in community-dwelling people with spinal cord dysfunction. Arch Phys Med Rehabil 2006; 87: 1305-1309.

15 Paterson DL, Bonomo RA. Extended-spectrum beta-lactamases: a clinical update. Clin Microbiol Rev 2005; 18: 657-686.

16 Lautenbach E, Patel JB, Bilker WB, Edelstein PH, Fishman NO. Extended-spectrum beta-lactamase-producing Escherichia coli and Klebsiella pneumoniae: risk factors for infection and impact of resistance on outcomes. Clin Infect Dis 2001; 32: 1162-1171.

17 Stamm WE, Hooton TM. Management of urinary tract infections in adults. N Engl J Med 1993; 329: 1328-1334.

18 Huh K, Kim J, Cho SY, Ha YE, Joo EJ, Kang Cl et al. Continuous increase of the antimicrobial resistance among gram-negative pathogens causing bacteremia: a nationwide surveillance study by the Korean Network for Study on Infectious Diseases (KONSID). Diagn Microbiol Infect Dis 2013; 76: 477-482.

19 Stover SL, Lloyd LK, Waites KB, Jackson AB. Urinary tract infection in spinal cord injury. Arch Phys Med Rehabil 1989; 70: 47-54.

20 Pannek J. Treatment of urinary tract infection in persons with spinal cord injury: guidelines, evidence, and clinical practice. A questionnaire-based survey and review of the literature. J Spinal Cord Med 2011; 34: 11-15. 